\title{
STELLAR AND TOTAL BARYON MASS FRACTIONS IN GROUPS AND CLUSTERS SINCE REDSHIFT 1*
}

\author{
S. Giodini ${ }^{1}$, D. Pierini ${ }^{1}$, A. Finoguenov ${ }^{1,2}$, G. W. Pratt $^{1}$, H. Boehringer ${ }^{1}$, A. Leauthaud ${ }^{3}$, L. Guzzo ${ }^{4}$, H. Aussel ${ }^{5}$,

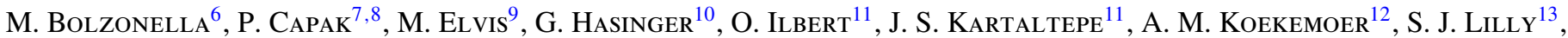 \\ R. Massey ${ }^{8}$, H. J. McCracken ${ }^{14}$, J. Rhodes ${ }^{8,15}$, M. Salvato ${ }^{8}$, D. B. SAnders ${ }^{11}$, N. Z. Scoville ${ }^{7}$, S. Sasaki ${ }^{16,17}$, V. Smolcic ${ }^{8}$, \\ Y. TANIGUCHI ${ }^{18}$, D. ThOMPSON ${ }^{8,19}$ AND THE COSMOS COLLABORATION \\ ${ }^{1}$ Max Planck Institut für Extraterrestrische Physik, Giessenbachstrasse, Garching bei München D-85748, Germany \\ ${ }^{2}$ University of Maryland, Baltimore County, 1000 Hilltop Circle, Baltimore, MD 21250, USA \\ ${ }^{3}$ LBNL \& Berkeley Center for Cosmological Physics, University of California, Berkeley, CA 94720, USA \\ ${ }^{4}$ INAF-Osservatorio Astronomico di Brera, Via Bianchi 46, I-23807 Merate (LC), Italy \\ 5 AIM Unité Mixte de Recherche CEA CNRS, Université Paris VII UMR n158, Paris, France \\ ${ }^{6}$ INAF_-Bologna Astronomical Observatory, via Ranzani 1, I-40127 Bologna, Italy \\ ${ }^{7}$ Spitzer Science Center, 314-6 Caltech, Pasadena, CA 91125, USA \\ ${ }^{8}$ California Institute of Technology, MC 105-24, 1200 East California Boulevard, Pasadena, CA 91125, USA \\ ${ }^{9}$ Harvard-Smithsonian Center for Astrophysics, 60 Garden Street, Cambridge, MA 02138, USA \\ ${ }^{10}$ Max-Planck-Institut für Plasmaphysik, Boltzmannstrasse 2, Garching bei München D-85748, Germany \\ ${ }^{11}$ Institute for Astronomy, University of Hawaii, 2680 Woodlawn Drive, Honolulu, HI 96822, USA \\ ${ }^{12}$ Space Telescope Science Institute, 3700 San Martin Drive, Baltimore, MD 21218, USA \\ ${ }^{13}$ Institute of Astronomy, Department of Physics, Eidgenössische Technische Hochschule, ETH Zurich, CH-8093, Switzerland \\ ${ }^{14}$ Institut d'Astrophysique de Paris, UMR 7095 CNRS, Université Pierre et Marie Curie, 98 bis boulevard Arago, 75014 Paris, France \\ 15 Jet Propulsion Laboratory, California Institute of Technology, Pasadena, CA 91109, USA \\ ${ }^{16}$ Astronomical Institute, Graduate School of Science, Tohoku University, Aramaki, Aoba, Sendai 980-8578, Japan \\ 17 Physics Department, Graduate School of Science and Engineering, Ehime University, 2-5 Bunkyo-cho, Matsuyama 790-8577, Japan \\ ${ }^{18}$ Research Center for Space and Cosmic Evolution, Ehime University, 2-5 Bunkyo-cho, Matsuyama 790-8577, Japan \\ ${ }^{19}$ Large Binocular Telescope Observatory, University of Arizona, Tucson, AZ 85721, USA \\ Received 2008 December 16; accepted 2009 July 21; published 2009 September 3
}

\begin{abstract}
We investigate if the discrepancy between estimates of the total baryon mass fraction obtained from observations of the cosmic microwave background (CMB) and of galaxy groups/clusters persists when a large sample of groups is considered. To this purpose, 91 candidate X-ray groups/poor clusters at redshift $0.1 \leqslant z \leqslant 1$ are selected from the COSMOS $2 \mathrm{deg}^{2}$ survey, based only on their X-ray luminosity and extent. This sample is complemented by 27 nearby clusters with a robust, analogous determination of the total and stellar mass inside $R_{500}$. The total sample of 118 groups and clusters with $z \leqslant 1$ spans a range in $M_{500}$ of $\sim 10^{13}-10^{15} M_{\odot}$. We find that the stellar mass fraction associated with galaxies at $R_{500}$ decreases with increasing total mass as $M_{500}^{-0.37 \pm 0.04}$, independent of redshift. Estimating the total gas mass fraction from a recently derived, highquality scaling relation, the total baryon mass fraction $\left(f_{500}^{\text {stars }+ \text { gas }}=f_{500}^{\text {stars }}+f_{500}^{\text {gas }}\right)$ is found to increase by $\sim 25 \%$, when $M_{500}$ increases from $\langle M\rangle=5 \times 10^{13} M_{\odot}$ to $\langle M\rangle=7 \times 10^{14} M_{\odot}$. After consideration of a plausible contribution due to intracluster light (11\%-22\% of the total stellar mass) and gas depletion through the hierarchical assembly process (10\% of the gas mass), the estimated values of the total baryon mass fraction are still lower than the latest CMB measure of the same quantity (WMAP5), at a significance level of $3.3 \sigma$ for groups of $\langle M\rangle=5 \times 10^{13} M_{\odot}$. The discrepancy decreases toward higher total masses, such that it is $1 \sigma$ at $\langle M\rangle=7 \times 10^{14} M_{\odot}$. We discuss this result in terms of nongravitational processes such as feedback and filamentary heating.
\end{abstract}

Key words: cosmological parameters - cosmology: observations - diffuse radiation - galaxies: clusters: general galaxies: stellar content $-\mathrm{X}$-rays: galaxies: clusters

\section{INTRODUCTION}

The baryon mass fraction is a parameter which can be constrained by the primordial light element abundance set by the nucleosynthesis at very early times. It has been measured to a very high precision from the five years Wilkinson Microwave Anisotropy Probe (WMAP5) observations of the cosmic microwave background (CMB), giving a value of

\footnotetext{
* Based on observations obtained with XMM-Newton, an ESA science mission with instruments and contributions directly funded by ESA Member States and NASA; also based on data collected at: the NASA/ESA Hubble Space Telescope, obtained at the Space Telescope Science Institute, which is operated by AURA Inc., under NASA contract NAS 5-26555, the Subaru Telescope, which is operated by the National Astronomical Observatory of Japan, the European Southern Observatory, Chile, under Large Program 175.A-0839, and the Canada-France-Hawaii Telescope operated by the National Research Council of Canada, the Centre National de la Recherche Scientifique de France and the University of Hawaii.
}

$f_{\mathrm{b}}^{\text {WMAP5 }}=0.171 \pm 0.009$ (Dunkley et al. 2009)..$^{20}$ An independent measure of this quantity can also be achieved with galaxy clusters. These structures are large enough to be representative of the baryon content of the universe, which exists mainly in the form of X-ray-emitting gas and stars. In the absence of dissipation, they are expected to provide a baryon mass fraction $\mathrm{f}_{b}$ comparable to the one measured from the CMB (White et al. 1993; Evrard 1997).

Galaxy systems appear in a wide range of masses, from $\sim 10^{13}$ to $\sim 10^{15} M_{\odot}$. In a hierarchical scenario, (White \& Frenk 1991) the less massive ones, $\left(M<10^{14} M_{\odot}\right.$, referred as groups) are the building blocks for the most massive ones (clusters). However, the vast majority of the attempts to estimate the baryon mass

\footnotetext{
${ }^{20}$ When the WMAP5 data are combined with the distance measurements from the Type Ia supernovae (SN) and the Baryon Acoustic Oscillations (BAO), $f_{\mathrm{b}}=\Omega_{\mathrm{b}} / \Omega_{\mathrm{m}}=0.1654 \pm 0.0062$ (Komatsu et al. 2009).
} 
fraction in nearby clusters have reported smaller values than expected (Ettori 2003; Lin et al. 2003; Biviano \& Salucci 2006; McCarthy et al. 2007). In addition, this discrepancy appears to be larger for groups than for clusters (Lin et al. 2003). Explanations for this invoke physical processes which lower $f_{\mathrm{b}}$ in clusters relative to the universal fraction (see, e.g., Bialek et al. 2001; He et al. 2006), baryon components that fail detection by standard X-ray and/or optical techniques (see Ettori 2003; Lin \& Mohr 2004), or a systematic underestimate of $\Omega_{\mathrm{m}}$ by WMAP (McCarthy et al. 2007).

McCarthy et al. (2007) extensively discuss possible explanations for the missing baryons. They concluded that the observed stellar mass function limits the contribution by low-mass stars and brown dwarfs to a negligible fraction of the total stellar mass; furthermore they rule out a contribution by large amounts of centrally concentrated gas, on the basis of inconsistencies with current X-ray data and the assumption of hydrostatic equilibrium. Consideration of the so-called intracluster light (ICL) results into a discrepancy at the $3.2 \sigma$ level with respect to WMAP3 across the mass range $6 \times 10^{13}-10^{15} M_{\odot}$ (Gonzalez et al. 2007). As discussed by these authors, systematics may help reconciling their results with the WMAP estimate.

In this respect, the correct determination of the gas mass fraction may be crucial. In fact, studies of the individual baryon components (stars associated with galaxies and gas) have shown that the stellar $\left(f_{500}^{\text {stars }}=M_{500}^{\text {stars }} / M_{500}\right)$ and gas mass fractions within $R_{500}{ }^{21}\left(f_{500}^{\text {gas }}=M_{500}^{\text {gas }} / M_{500}\right)$ exhibit opposite behaviors as a function of the total system mass. In particular, clusters have a higher gas mass fraction than groups (Vikhlinin et al. 2006; Arnaud et al. 2007; Sun et al. 2009), but a lower stellar mass fraction (Lin et al. 2003). This has been interpreted as a difference in the star formation efficiency between groups and clusters (David et al. 1990; Lin et al. 2003; Laganá et al. 2008).

On the other hand, the mass dependence of the gas fraction and the discrepancy between the baryon mass fraction in groups/ clusters and the WMAP value can be understood in terms of nongravitational processes. In fact active galactic nucleus (AGN)-heating (which can drive the gas outside the potential well) or gas pre-heating (which inhibits the gas from falling toward the center of the potential) can explain the lack of gas within $r_{500}$ in groups. Therefore, groups appear as the critical systems to assess the universality of the baryon fraction, and to understand complex physical processes affecting both the gas and the stellar components.

Little work has been conducted on estimation of the baryon mass fraction at the group regime, mainly because of the lack of groups in existing catalogs and the difficulty of estimating masses for the individual components and the total. An insufficient sampling of the range in total mass spanned by groups and clusters is problematic for studying their overall properties in terms of mean and scatter of the population. ${ }^{22}$ A galaxy group/ cluster is the result of the assembly history of the dark matter halo, as well as of the star formation processes affecting the gas. Both processes lead to multivariate outcomes and produce a large intrinsic scatter in the distribution of the observed properties of groups and clusters. Therefore, it is essential to have a large enough sample to be representative of the population, and

\footnotetext{
$21 R_{\Delta}(\Delta=500,200,2500)$ is the radius within which the mass density of a group/cluster is equal to $\Delta$ times the critical density $\left(\rho_{c}\right)$ of the universe. Correspondingly, $M_{\Delta}=\Delta \rho_{c}(z)(4 \pi / 3) R_{\Delta}^{3}$ is the mass inside $R_{\Delta}$.

22 The conclusions of Lin et al. (2003) and Gonzalez et al. (2007), for example, are based only on, respectively, 27 and 23 systems, but only 3 and 5 of them are less massive than $10^{14} h^{-1} M_{\odot}$.
}

unbiased by selection effects, to be able to investigate the mean trend precisely.

Once such a sample is available, interesting questions to address are: (1) how does the stellar mass fraction behave across the total range of masses?; (2) does the relation between the stellar mass fraction and the total system mass evolve with redshift?; (3) how does the gas mass fraction change as a function of the system total mass?; (4) is the total baryonic fraction in groups/ clusters of galaxies consistent with the WMAP5 value?

In this paper, we select the currently largest X-ray-selected sample of groups from the COSMOS $2 \mathrm{deg}^{2}$ survey which consists of 91 high-quality systems at $0.1 \leqslant z \leqslant 1$. Existing observations currently do not give constraints on the evolution of the baryonic components in individual systems at $z \geqslant 0.1$. Our data allow us to put constraints on the redshift evolution of the average stellar fraction with mass, which we find to be consistent with zero (Section 4.2). Observational constraints on the evolution of the average gas mass fraction also suggest zero evolution in the cluster regime (Allen et al. 2004). We assume that this is applicable to our groups in the absence of observations to the contrary and we note that simulations support this hypothesis (Kravtsov et al. 2005).

We complement our sample with 27 nearby clusters investigated by Lin et al. (2003) in order to achieve a span of 2 orders of magnitude in total mass $\left(10^{13}<M<10^{15} M_{\odot}\right)$. In Section 3, the total mass of stars associated with galaxies is directly determined for each group, and we investigate the relation between the stellar mass fraction and the total mass of the system. In Section 4, we combine the stellar mass fraction estimates with the most recent determination of the relation between gas mass fraction and total mass based on a compilation of 41 local $(z \leqslant 0.2)$ X-ray groups and clusters, spanning the same range in mass as ours (Pratt et al. 2009), and we compute the total baryon fraction. We discuss results in Section 5.

We adopt a $\Lambda$ CDM cosmological model $\left(\Omega_{\mathrm{m}}=0.258\right.$, $\Omega_{\Lambda}=0.742$ ) with $\mathrm{H}_{0}=72 \mathrm{~km} \mathrm{~s}^{-1} \mathrm{Mpc}^{-1}$, consistently with WMAP5 (Dunkley et al. 2009; Komatsu et al. 2009). Unless otherwise stated all quantities are estimated at an overdensity of 500 .

\section{THE SAMPLE}

\subsection{The COSMOS Survey of Groups/Poor Clusters}

The Cosmic Evolution Survey (COSMOS; Scoville et al. 2007a) was designed to probe how galaxies, AGNs, and dark matter evolve together within the large-scale structure. The survey is based on multiwavelength imaging and spectroscopy from X-ray to radio wavelengths and covers a $2 \mathrm{deg}^{2}$ area, including Hubble Space Telescope (HST) imaging of the entire field (Koekemoer et al. 2007). Large-scale structures in the COSMOS field have been characterized in terms of galaxy overdensity using photometric redshifts (Scoville et al. 2007b), weak lensing convergence maps (Massey et al. 2007), diffuse X-ray emission (Finoguenov et al. 2007), and a combination of these (Guzzo et al. 2007). In particular, the entire COSMOS region was imaged through 54 overlapping XMM-Newton pointings (1.5 Ms; Hasinger et al. 2007). Additional Chandra observations (1.8 Ms; Elvis et al. 2006) mapped the central region to higher resolution.

In this study, we use X-ray detection, gravitational lensing signal, optical photometric, and spectroscopic data of the clusters and groups identified in the COSMOS survey. The $\mathrm{X}$-ray data reduction is described in detail in Finoguenov et al. 


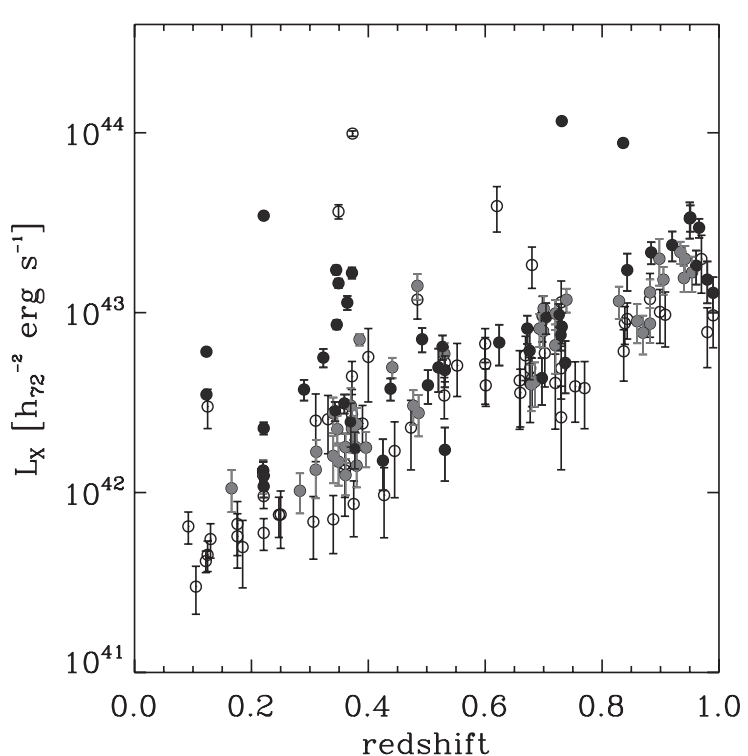

Figure 1. Rest-frame 0.1-2.4 keV luminosity vs. redshift for the 151 COSMOS candidate X-ray groups/clusters at $0.1<z<1$.0. Filled circles mark the 91 objects considered in this study: dark or light gray identifies objects with flag 1 (45) or 2 (46), respectively.

(2007) and A. Finoguenov et al. (2009, in preparation). From a composite mosaic of the XMM-Newton and Chandra X-ray data, it has been possible to detect and measure the flux of extended sources (i.e., groups and clusters) down to a limit of $10^{-15} \mathrm{erg} \mathrm{s}^{-1} \mathrm{~cm}^{-2}$, as described in the corresponding catalog (A. Finoguenov et al. 2009, in preparation). Extended source detection was based on a wavelet scale-wise reconstruction of the image, as described in Vikhlinin et al. (1998b), employing angular scales from $8^{\prime \prime}$ to 2 '.1. Clusters and groups of galaxies were effectively selected by the spatial extent of their X-ray emission, following the approach of Rosati et al. (1998), Vikhlinin et al. (1998b), and Moretti et al. (2004). The cluster detection algorithm consists of: (1) removal of the background; (2) detection of AGN; (3) removal of AGN flux from large scale; and (4) search for extended emission. As a result, a total of 219 $\mathrm{X}$-ray extended sources were identified in the redshift range $0<z<1.6$; they span the rest-frame $0.1-2.4 \mathrm{keV}$ luminosity range $10^{41} \leqslant L_{X} \leqslant 10^{44} \mathrm{erg} \mathrm{s}^{-1}$, which is typically populated by groups and poor clusters.

Quality flags tag individual systems. Flag 1 is assigned to objects whose center corresponds to the X-ray peak of the source, while flag 2 objects have their spectral extraction region redefined to include only their robust association with a unique optical system. A redshift was assigned to each candidate X-ray group/cluster, corresponding to the mean of the photometric redshift (photo- $z$ ) distribution of the red-sequence galaxies as identified in Tanaka et al. (2009, in preparation), if present, and lying within the X-ray overdensity contour region. This redshift is checked against the available spectroscopic redshifts mostly provided by the $z$ COSMOS spectroscopic survey (Lilly et al. 2007). The presence of a red sequence is not required for the group/cluster detection: if no overdensity of red sequence galaxies is found in the photo- $z$ space, the spectroscopic data only are checked for the presence of a galaxy overdensity in the same area. Flag 3 is assigned to high $z(z>1)$, not spectroscopically confirmed candidate groups. Flag 4 is assigned when multiple optical counterparts are present within the X-ray overdensity contour region. In this study, only systems with quality flag 1 or 2 are considered.
The galaxy-group detection is irrespective of any optical characteristic, being based only on the presence of an $\mathrm{X}$-ray extended source. The $\mathrm{X}$-ray selection is an approximate selection by halo mass, due to the tight X-ray luminosity-mass relation (Pratt et al. 2009); in this regard, our selection is thus unbiased with respect to both the optical properties of the groups in our sample and the X-ray characteristic of the systems.

The purposes of the present study lead us to introduce three further selection criteria: (1) only candidate groups/clusters detected in X-rays with a significance higher than $3 \sigma$ on the flux determination are considered. Selection of the most robust candidates minimizes contamination by loose galaxy aggregations or superposition of AGN along the line of sight. (2) Only Xray extended sources with $L_{\mathrm{X}}>10^{42} \mathrm{erg} \mathrm{s}^{-1}$ are considered, in order to limit contamination from starburst galaxies (Grimm et al. 2003) or field elliptical galaxies with X-ray halos (Diehl \& Statler 2007). (3) We limit the redshift range to $0.1 \leqslant z \leqslant 1.0$, where photo- $z$ of individual galaxies are most robust (Ilbert et al. 2009); furthermore, in this range the quality of the photo- $z$ is equivalent to that of low-resolution spectroscopy.

Figure 1 reproduces the X-ray luminosity distribution as a function of redshift for the candidate X-ray groups/clusters within $z=1$ (151 out of 219 systems). The flag $1+2$ sample selected for this study contains 114 objects, of which 44 were present in Finoguenov et al. (2007). It contains only three systems at $z \leqslant 0.2$ (Figure 1 ), which is the redshift range covered by analogous studies on $f_{\mathrm{b}}$ in groups/clusters (Lin et al. 2003; Gonzalez et al. 2007). On the other hand, it contains systems with particularly low X-ray luminosities (i.e., with $10^{42}<L_{\mathrm{X}}<5 \times 10^{42} \mathrm{erg} \mathrm{s}^{-1}$ ), though only for $z<0.5$. The sample considered in this study is reduced to 91 objects after removal of 23 groups with unreliable estimates of the total stellar mass in galaxies (Section 3.2). Out of these 91 candidate groups/poor clusters, 51 are already spectroscopically confirmed (i.e., are associated with at least three galaxies with similar spectroscopic redshifts).

\subsection{COSMOS X-ray-Selected Groups/Poor Clusters: Total Mass Estimate}

In the original catalog (A. Finoguenov et al. 2009, in preparation), $M_{200}$ is computed using an $L_{X}-M_{200}$ relation established via the weak lensing analysis in A. Leauthaud et al. (2009, in preparation). Briefly, the COSMOS group sample is divided into nine bins that span the redshift range $0.1<z<0.9$ and with $10^{41.8}<L_{\mathrm{X}} / E(z)<10^{43.5} \mathrm{erg} \mathrm{s}^{-1}$, where the function $E(z) \equiv \sqrt[2]{\Omega_{\mathrm{m}}(1+z)^{3}+\Omega_{\Lambda}}$ represents the Hubble parameter evolution for a flat metric. Only systems with a clear, visually identified BCG are used for this analysis, to minimize issues due to incorrect centering. For each bin, the weak lensing signal is calculated from $r \sim 50 \mathrm{kpc}$ to $r \sim 3 \mathrm{Mpc}$ in logarithmically spaced radial bins. A weak lensing signal is detected all the way to $3 \mathrm{Mpc}$ ensuring that the lens density is probed well beyond the virial radius. The results are fit with a parametric model which is the sum of an Navarro-Frenk-White (NFW) profile (Navarro et al. 1997) and a point-source term due to the mass of the central BCG. The theoretical relation between mass and concentration from Zhao et al. (2008) has been used in the fit for the NFW component and the mean stellar mass of the central BCGs is used in order to scale the point-source term. A comparison between the relation obtained from the combination of the the COSMOS data and cluster data from Hoekstra (2007) is consistent with that obtained by Rykoff et al. (2008) based on Sloan Digital Sky Survey (SDSS) data. We adopt the 
following functional form for the $L_{\mathrm{X}}-M$ relation,

$$
\frac{M_{200} E(z)}{M_{0}}=A\left(\frac{L_{X} E(z)^{-1}}{L_{X, 0}}\right)^{\alpha},
$$

where $M_{0}=10^{13.7} M_{\odot}, L_{X, 0}=10^{42.7} \mathrm{erg} \mathrm{s}^{-1}$. Fitting only the COSMOS data yields the best-fit parameters $\log _{10}(A)=$ $0.106 \pm 0.053$ and $\alpha=0.56 \pm 0.12$ (cited errors are statistical only). Further details regarding the weak lensing analysis in COSMOS can be found in A. Leauthaud et al. (2009, in preparation).

The baryon fraction in groups/clusters can be studied at any radius, though it is desirable to study it at the largest radius possible with respect to the virial radius of the system because of the radial dependencies of the different components. The largest radius for which reliable X-ray hydrostatic masses are available is $R_{500}$ (e.g., Arnaud et al. 2005; Vikhlinin et al. 2006; Sun et al. 2009). Hereafter, we use $M_{500}$ instead of $M_{200}$, to enable a comparison at the same radius with previous studies on $f_{\mathrm{b}}$ in nearby groups/clusters. The catalog value of $M_{200}$ is converted into $M_{500}$ assuming an NFW profile with a constant concentration parameter $(c=5)$.

\subsection{COSMOS Galaxies: Multiwavelength Photometry and Photometric Redshifts}

The COSMOS area has been imaged in 30 bands including broad- (SUBARU, Taniguchi et al. 2007a; CFHT; H. J. McCracken et al. 2009, in preparation), medium-, and narrowbands (SUBARU; Y. Taniguchi et al. 2009, in preparation), ranging from the far-ultraviolet (GALEX; Zamojski et al. 2007) to the mid-infrared (Spitzer; Sanders et al. 2007). This multiwavelength data set is collected in a master photometric catalog. P. Capak et al. (2009, in preparation) discuss in detail source detection and extraction of photometry. The COSMOS photometric catalogue is complete down to a total $i$-band magnitude of 26.5 AB mag. Ilbert et al. (2009) and Salvato et al. (2009) computed highly reliable photometric redshifts with unprecedented accuracy for a survey this large, owing to the extraordinarily large number of photometric bands. Redshifts were attributed to individual galaxies via a standard $\chi^{2}$ fitting procedure (Arnouts et al. 2002) encoded in Le Phare, ${ }^{23}$ written by S. Arnouts and O. Ilbert. Best-fit solutions from this photo- $z$ algorithm were trained on a composite spectroscopic sample of objects brighter than $i_{\mathrm{AB}}=25$ (see Table 3 in Ilbert et al. 2009), mostly made of $\sim 4000$ bright galaxies (i.e., with $i_{\mathrm{AB}}<22.5$ ) observed as part of the $z$ COSMOS spectroscopic survey (Lilly et al. 2007). Comparison of photometric and spectroscopic redshifts gives a typical root-mean-square scatter of the photo-zs equal to $\sigma_{\text {photo }-z}=0.02 \times(1+z)$ for $i_{\mathrm{AB}} \leqslant 25$ and $z<1.25$ (Ilbert et al. 2009). In the presence of X-ray-emitting objects (AGNs), photometric redshifts were independently estimated by Salvato et al. (2009).

As a by-product of the photo- $z$ determination, spectroscopic types were attributed to individual galaxies on the basis of their best-fit broadband spectral energy distributions (SEDs). This information is used to estimate the stellar mass of a galaxy, which is obtained from the conversion of the $K s$-band luminosity (Ilbert et al. 2009) using an evolving galaxy-type dependent stellar mass-to- $K s$-band luminosity ratio $M / L_{\mathrm{Ks}}$ (Arnouts et al. 2007). This relation has been established using a Salpeter initial mass function (Salpeter 1955). Stellar masses of individual galaxies are contained in the COSMOS photometric catalogue; the fractional error on the stellar mass of a galaxy is typically equal to $34 \%$, and is dominated by the mean scatter on $M / L_{K s}$ (Arnouts et al. 2007).

This uncertainty pertains to the aforementioned method of estimating stellar masses. Individual galaxy stellar masses may differ by a factor 2-3, depending on the method used to estimate the mass (e.g., Longhetti \& Saracco 2009; Küpcü Yoldaş et al. 2007). This uncertainty is the product of several factors; it mostly reflects the range of assumptions in differing models as for the star formation history (e.g., single burst versus multiple bursts versus continuum star formation activity) and the attenuation of stellar light by dust (e.g., starburst-like versus normal star-forming disk like). In addition, it results from different implementations of complex physics, such as the asymptotic-giant-branch phase of stellar evolution and metal enrichment). This scatter does not reflect the uncertainty of the present method, which is $34 \%$ for individual galaxies as detailed above. This latter value is the uncertainty we attribute to individual galaxy stellar masses in the present study.

\subsection{Nearby Clusters}

The COSMOS sample is mostly composed of groups. Therefore, we complement it with a sample of 27 nearby X-rayselected clusters with sufficiently deep 2MASS photometry (Lin et al. 2003, LMS03) to estimate accurate stellar masses. The total and stellar masses were derived by LMS03 in a manner consistent with ours. In particular, the total cluster mass is estimated from an $M_{500}-T_{\mathrm{X}}$ relation. The stellar masses are estimated from the total $K$ band luminosity of each cluster, assuming an average stellar mass-to-light ratio (M/L) which takes into account the varying spiral galaxy fraction as a function of the cluster temperature.

LMS03 provide estimates of the total gas fraction obtained from either X-ray data or from a scaling relation; we use instead the most recent scaling relations of Pratt et al. (2009), based on hydrostatic mass estimates, in order to reduce systematic effects. We apply this both to our sample and the one of LMS03.

\section{DATA ANALYSIS}

\subsection{Galaxy Stellar Mass Function: Completeness and Extrapolation}

The low-mass end of the galaxy stellar mass function of the individual COSMOS groups/poor clusters is probed to different extents by observations, since these systems span a rather large redshift range $(0.1 \leqslant z \leqslant 1)$. In order to achieve a common footing, the completeness in galaxy absolute magnitude (stellar mass) of the sample must be understood.

First, we divide the sample into two redshift bins $(0.1-0.5$ and $0.5-1.0)$ containing a similar number of objects, since the cosmic stellar mass density is observed to drop by a factor of 2 from $z \sim 0$ to 1 in the field (Wilkins et al. 2008, and references therein). The completeness mass is estimated at $z=0.5$ and $z=1.0$ from a fit of its behavior as a function of redshift, obtained using a sampling of 0.1 in redshift as follows (Bolzonella et al. 2009). Firstly, we derive the stellar mass ( $\left.M_{\mathrm{lim}}\right)$ that each object would have if its apparent magnitude was equal to the sample limit magnitude (i.e. $i_{A B}=25$ ), viz.,

$$
\log M_{\lim }=\log M+0.4 \times\left(i_{A B}-25.0\right),
$$

\footnotetext{
23 www.lam.oamp.fr/arnouts/LE_PHARE.html
} 


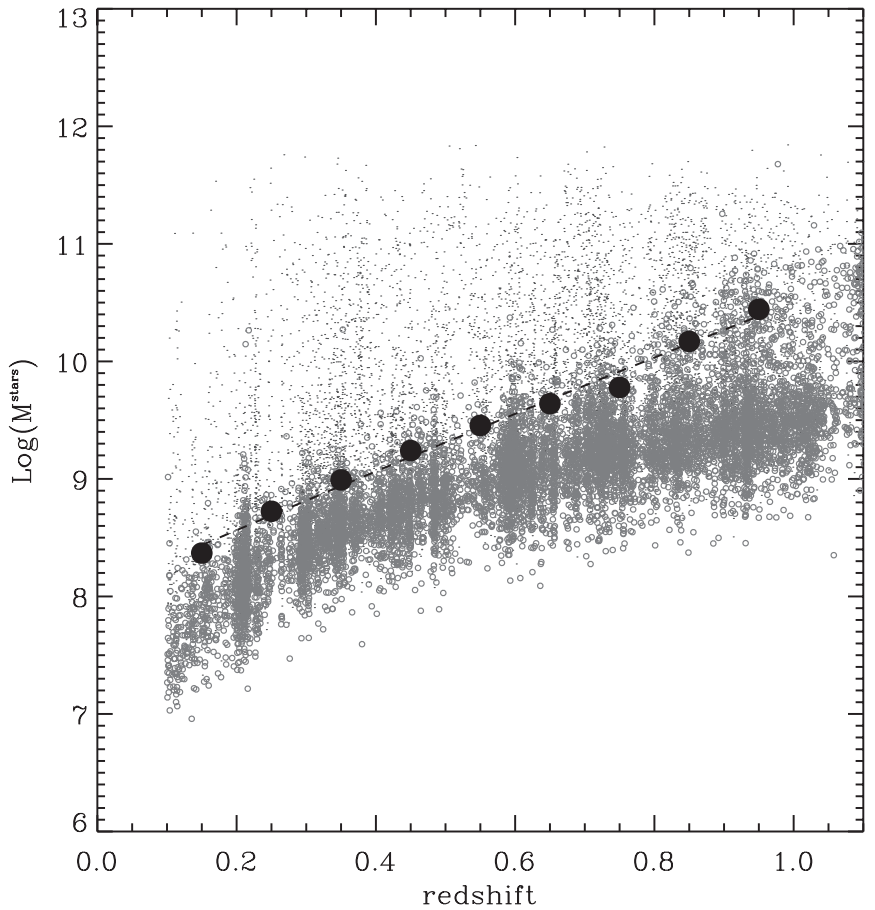

Figure 2. Completeness stellar mass for our sample is computed from the fit (black dashed line) to the $95 \%$ percentile of the distribution in $M_{\text {lim }}$ (see text) for galaxies in the $20 \%$ lower percentile in magnitude (gray circles) as a function of redshift. The black dots represent the stellar masses for all galaxies with $i_{A B} \leqslant 25$. To reduce the plot size, we plot only one point in 10 .

where $M$ is the stellar mass of a galaxy with apparent magnitude $i_{\mathrm{AB}}$. Second, we derive the $95 \%$ percentile of the distribution in $M_{\text {lim }}$ for galaxies in the lower $20 \%$ percentile in magnitude (i.e., $\left.i_{A B} \geqslant 23.6\right)$ in each bin of 0.1 in redshift. Finally, a fit to the corresponding envelope as a function of redshift is performed for $0.1 \leqslant z \leqslant 1.0$; the ensuing values represent the stellar mass completeness as a function of redshift for our sample. Figure 2 illustrates the behavior of the stellar mass completeness as a function of redshift.

For instance, the stellar mass completeness at $z=1$ $\left(M_{\text {compl }}=10^{10.4} M_{\odot}\right)$ is about an order of magnitude lower than the so-called transition stellar mass at $z \leqslant 1$ (e.g., Bundy et al. 2005; Pannella et al. 2006). This confirms that a rich mixture of morphologies and, thus, star formation histories (Sandage 1986) is present among the member galaxies of the COSMOS $\mathrm{X}$-ray-selected groups/poor clusters.

We compute the total stellar mass associated with galaxies of a given system as follows. We first add the stellar masses of galaxies more massive than the completeness mass (at $z=0.5$ or 1 ) for which membership to a given group/poor cluster is determined (as described in Section 3.2.1). Taking into account the mass of the individual galaxies, rather than their statistical distribution (as in Lin et al. 2003), becomes increasingly important for groups, where the BCG is a large fraction of the total stellar mass.

The contribution from less massive galaxies is estimated in a statistical manner from the composite stellar mass function (S. Giodini et al. 2009, in preparation), which can be robustly obtained only within two broad redshift bins $(0.1 \leqslant z \leqslant 0.5$ and $0.5<z \leqslant 1.0$ ). The stacked stellar mass function for systems falling in each redshift bin is fitted with a single Schechter function (Schechter 1976); the correction factor for stellar masses lower than the completeness mass, down to $\sim 10^{8} \mathrm{M}_{\odot}$ (typical mass of a dwarf galaxy), is given by:

$$
1-\frac{\int_{M_{\mathrm{compl}}}^{10^{13}} f(M) \cdot M d M}{\int_{10^{8}}^{10^{13}} f(M) \cdot d M},
$$

where $M_{\text {compl }}$ is the completeness mass for the given redshift range. The fractional contribution to the total stellar mass budget of galaxies with $10^{8} M_{\odot} \leqslant M \leqslant M_{\text {compl }}$ corresponds to $\sim 9 \%$ $(\sim 1 \%)$ at redshifts $0.5-1.0(0.1-0.5)$. These values are almost negligible, as in Lin et al. (2003), which confirms that the total stellar mass associated with galaxies can be achieved almost directly from the data for our sample of X-ray-selected groups / poor clusters at $0.1 \leqslant z \leqslant 1$.

\subsection{Total Stellar Mass (in Galaxies) \\ 3.2.1. Statistical Membership}

As a first step, we estimate a projected total stellar mass, which is the sum of the stellar masses of all potential member galaxies down to the completeness mass of either redshift bin to which a group belongs (i.e., $0.1-0.5$ or $0.5-1.0$ ). Candidate members are defined as all the galaxies within a projected distance equal to $R_{500}$ from the X-ray centroid of a group/ poor cluster and within $0.02 \times(1+z)$ from its redshift (given in the X-ray catalog). Then we perform a foreground/background correction by measuring the total stellar mass of galaxies contained in 20 circular areas which have the same radius as $R_{500}$ and have photometric redshifts consistent with that of a given system within the errors. These areas do not overlap either with the group or with other groups at the same redshift and are chosen to represent the coeval field environment. Field galaxies are selected in redshift and stellar mass following the same criteria as for the selection of potential member galaxies previously described. The mean and the standard deviation of the distribution of the total stellar masses computed in the 20 regions are taken as the value of the stellar mass associated with the foreground/background and its uncertainty, respectively. Finally, the foreground/background value is subtracted from the initial estimate of the total stellar mass of the system.

If the error on the foreground/background value is larger than half of the estimated total stellar mass content in galaxies of a given system, this system is removed from the sample. Obviously, a system is excluded also if the foreground/background correction exceeds the estimated total stellar mass content in galaxies. The variance on the total stellar mass budget in galaxies for a system is given by the sum in quadrature of the background uncertainty and the error on the total stellar mass of the galaxies of the system.

Furthermore, we checked the influence of masked areas on the reliability of the computed total stellar masses of individual groups. A region of the COSMOS area is masked when the image quality is poor owing to different reasons (e.g., field boundary, saturated stars, satellite tracks, and image defects). For galaxies with elliptical-like SEDs reliable photo$z \mathrm{~s}$ can be determined also in masked areas; therefore early-type galaxies falling in masked areas are considered. On average, the contribution of these objects to the stellar mass budget of a group is not expected to be negligible. In fact, in 30 out of 37 cases where early-type galaxies falling in masked areas are retrieved, the new stellar mass fraction (computed in Section 4.1) is consistent with that of other groups with the same $M_{500}$, whatever the redshift. Conversely, late-type galaxies falling in masked areas are not considered and the impact of this choice 
is tested a posteriori. For 23 out of 114 groups, the number of statistically established member galaxies is less than six and the total stellar mass is systematically lower than the mean for groups of similar total masses, irrespective of $M_{500} .{ }^{24}$ These 23 objects span the entire total mass range and their exclusion does not affect our results on the stellar mass fraction; at the same time, the scatter in the stellar mass fraction decreases by $30 \%{ }^{25}$ Only the resulting sample of 91 galaxy systems with at least six members, spanning 2 orders of magnitude in X-ray luminosity, is considered in the following analysis; hereafter it is designated the COSMOS X-ray selected group sample.

\subsubsection{Deprojection}

The total stellar mass in galaxies, so far estimated, refers to a cylindrical section of the system projected onto the plane perpendicular to the line of sight. We therefore need to deproject the total stellar mass from two to three dimensions. The average galaxy distribution is described by a projected NFW profile in two dimensions (Bartelmann 1996; Navarro et al. 1997),

$$
\Sigma(x)=\frac{2 \rho_{\mathrm{s}} r_{\mathrm{s}}}{x^{2}-1} f(x),
$$

where

$$
f(x)= \begin{cases}1-\frac{2}{\sqrt{x^{2}-1}} \arctan \sqrt{\frac{x-1}{x+1}} & (x>1) \\ 1-\frac{2}{\sqrt{1-x^{2}}} \operatorname{arctanh} \sqrt{\frac{1-x}{1+x}} & (x<1) \\ 0 & (x=1)\end{cases}
$$

and as a generalized NFW profile in three dimensions

$$
\rho(x)=\frac{\rho_{\mathrm{s}}}{x(1+x)^{2}} .
$$

In both equations, the radial coordinate $x$ is the radius in units of a scale radius $r_{\mathrm{s}}, x \equiv r / r_{\mathrm{s}}$. The scale radius corresponds to the ratio between $R_{200}$ and the concentration parameter $c$ for the system. An average profile is produced using all 91 systems in our final sample, with a central density normalized to the number of groups. This high signal-to-noise, average two-dimensional galaxy distribution is best fitted by a two-dimensional NFW profile, where $r_{\mathrm{s}}=0.27 R_{200}$. The average radial profile is shown in Figure 3 together with its best fit (with a reduced $\chi^{2}$ value equal to 1.2). We remark that our aim is not to compute the concentration parameter of the galaxy distribution for individual systems, otherwise we should take into account the scatter in the evolution of the concentration parameter as a function of redshift. Instead, we want to compute an average correction for projection of the mass profile of a system as calculated in Section 3.2.1.

Using the best-fit values, we compute correction factors by integrating the average profile out to $R_{500}$,

$$
d p f=\frac{\int_{0}^{R_{500}} \rho(r) \cdot 4 \pi r^{2} d r}{\int_{0}^{R_{500}} \Sigma(r) \cdot 2 \pi r d r} .
$$

\footnotetext{
24 This tells that five members only are insufficient to determine the stellar mass budget of a group. In fact, when the total stellar mass or luminosity of a system is computed from a population of discrete sources, the scatter in the ensuing value turns out to be non linear when the number of discrete sources becomes small (e.g., of order 10 or less), as demonstrated by Gilfanov et al. (2004) in an analogous application.

${ }^{25}$ Nevertheless, these objects are potentially an interesting subpopulation characterized by an extremely slow build-up of stellar mass. Further, optical follow up will help to better assess their properties.
}

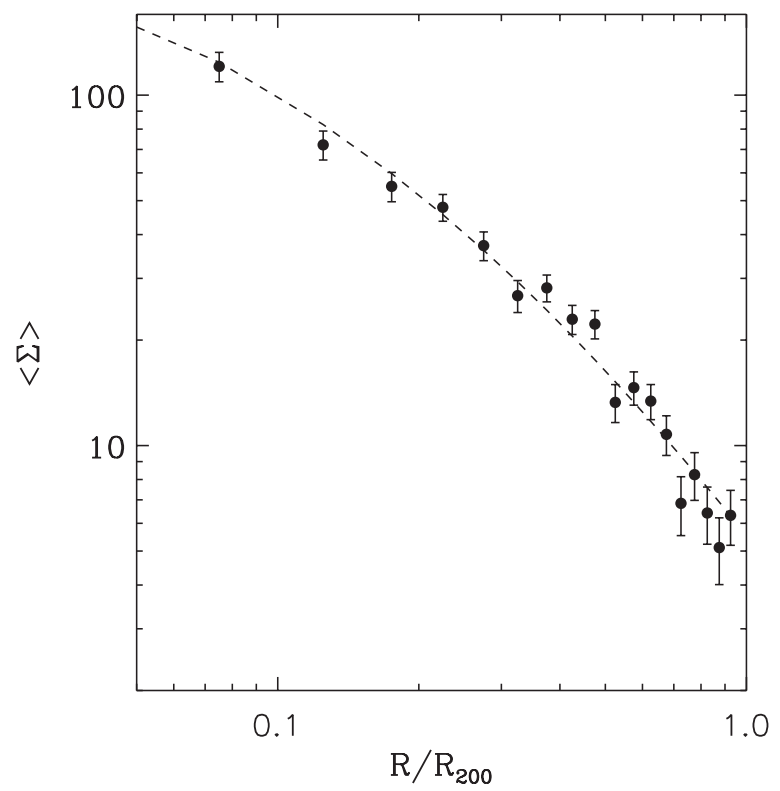

Figure 3. Radial profile of the average number galaxy density for the 91 COSMOS groups/poor clusters. The dashed line shows the best fit NFW profile $(c \sim 4)$. The unit of the surface density is number per area in unit of $\pi R_{200}^{2}$ and normalized to the total number of systems.

The deprojected total stellar mass of a system is then given by

$$
M_{500}^{\text {stars }}=d p f \times M_{\text {proj, } 500}^{\text {stars }},
$$

where $d p f=0.86$ is the correction factor.

\section{RESULTS}

\subsection{Stellar Mass Budget (Galaxy Component)}

Figure 4 shows the behavior of the total (deprojected) stellar mass in galaxies within $R_{500}, M_{500}^{\text {stars }}$, as a function of the total mass $M_{500}$ for the 91 COSMOS X-ray-selected groups. The distribution in Figure 4 exhibits a rather well defined trend, although a large scatter is present, especially at low masses, where values can range by a factor of 10 at a fixed total mass. Part of this large scatter may have a physical origin: different merging histories produce different total $\mathrm{M} / \mathrm{Ls}$ for fixed total assembled mass (cf. Sales et al. 2007).

We fit the relation between total stellar mass in galaxies and total mass for all 91 systems and for the 45 flag $=1$ groups only. Since the distribution in Figure 4 exhibits an intrinsic scatter larger than the errors on the individual points, the fit is performed using the weighted least square with intrinsic scatter (WLSS) method discussed in Pratt et al. (2006). This algorithm takes into account uncertainties on both stellar mass and total mass and the presence of intrinsic scatter in the data. There is a robust correlation between $M_{500}^{\text {stars }}$ and $M_{500}$ in the COSMOS $\mathrm{X}$-ray-selected groups

$$
M_{500}^{\text {stars }}=(0.30 \pm 0.02) \times\left(\frac{M_{500}}{5 \times 10^{13} h_{72}^{-1}}\right)^{\alpha},
$$




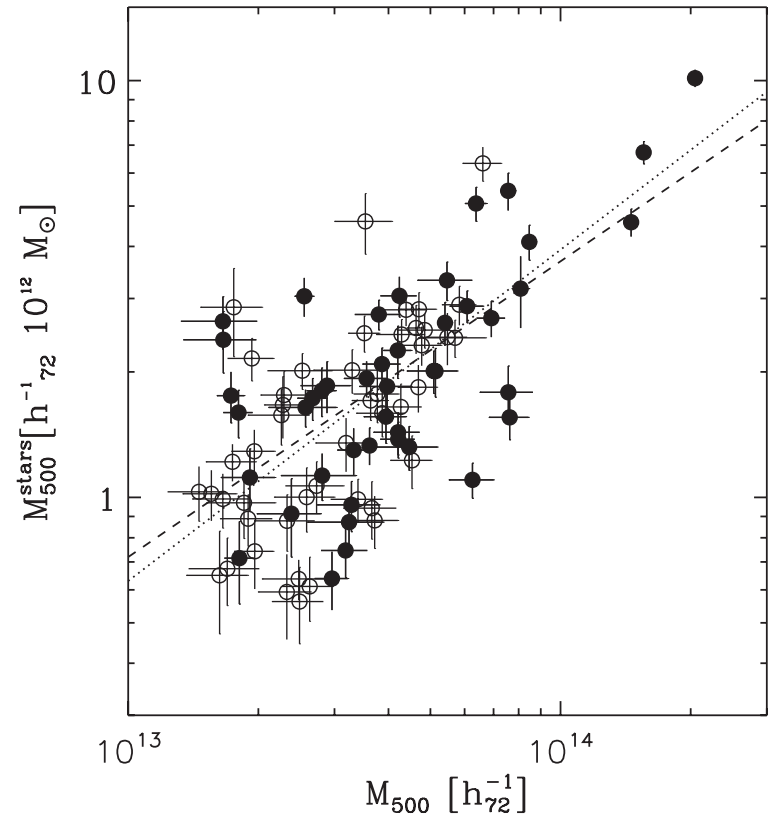

Figure 4. Total stellar mass in galaxies vs. total mass for the 91 COSMOS X-rayselected groups/poor clusters. Filled (empty) gray circles identify objects with flag $=1$ (2). The dashed (dotted) line represents the best fit relation derived for flag $=1$ (all) groups (see Equation (9)) derived taking into account uncertainties in both quantities and the intrinsic scatter of the relation.

where $\alpha=0.81 \pm 0.11$ for the entire sample and $\alpha=$ $0.72 \pm 0.13$ for the flag $=1$ subsample, and the (logarithmic) intrinsic scatter is equal to $35 \%$ in both cases ${ }^{26}$.

Fitting the stellar-to-total mass ratio versus total mass of the system for the full sample of COSMOS X-ray-selected groups only, we find

$$
f_{500}^{\mathrm{stars}}=5.0_{-0.1}^{+0.1} \times 10^{-2}\left(\frac{M_{500}}{5 \times 10^{13} M_{\odot}}\right)^{-0.26 \pm 0.09} .
$$

A fit to the Flag $=1$ sample gives equivalent results. Remarkably, the relation between the mass fraction of stars in galaxies and the total mass of the system for the COSMOS X-ray-selected groups is consistent within the errors with the one found in nearby clusters by LMS03 and Laganá et al. (2008). We now extend the range of total masses using the results from local clusters selected by LMS03, converting their measurements to our cosmology. Since these authors do not give the uncertainties associated with their total mass estimates, we assign a fixed fractional total mass uncertainty equivalent to the mean of that for the COSMOS groups $(\sim 30 \%)$. The best fit of the combined sample is

$$
f_{500}^{\mathrm{stars}}=5.0_{-0.1}^{+0.1} \times 10^{-2}\left(\frac{M_{500}}{5 \times 10^{13} M_{\odot}}\right)^{-0.37 \pm 0.04},
$$

with a typical logarithmic intrinsic scatter of $\sim 50 \%$. The data and best-fit relations are shown in Figure 5.

\footnotetext{
26 This result is robust against the presence of a pair of groups which are detected at the same redshift, but with a separation of the order of $R_{500}$. The two objects of this pair lie above the best-fit relation reproduced in Figure 4, perhaps as an effect of a bias in their estimated total stellar masses in galaxies. However, new fits performed after excluding these two groups give the same results as the previous ones.
}

To better elucidate trends with total mass, we divided the data set into five logarithmic bins of equal size in total mass, and computed the mean and standard deviation of the values of the mass fraction of stars in galaxies in each bin using the bi-weight estimators of Beers et al. (1990); they are relatively large, which gives a measure of the heterogeneity of the population. The large points with error bars show the trend of these binned data with total mass: there is good agreement with the best-fitting regression line to the unbinned points, as expected.

\subsection{Evolutionary Considerations}

Finally, we inspect the presence of evolution of the relation between $f_{500}^{\text {stars }}$ and $M_{500}$ by considering only systems at $z \leqslant 0.5$ (we cannot fit the relation for the high redshift systems since they do not cover a sufficient range in total mass). The ensuing fit is fully consistent with that obtained for the entire sample within the uncertainties.

We can put a constraint on the possible evolution of the relation by evaluating the change in the mean of $f_{\text {star }}$ for massive systems $\left(M_{500}>5 \times 10^{14} M_{\odot}\right)$ in two redshift bins $(z \leqslant 0.5$ and $z>0.5)$. The average $f_{\text {star }}$ changes from $0.031 \pm 0.013$ at $z \leqslant 0.5$ to $0.039 \pm 0.019$ at $z>0.5$, a less than $1 \sigma$ difference in mean values. Even taking the maximum distances between the two values given the uncertainties, the stellar mass fraction does not change by more than $35 \%$.

A second way to investigate a possible evolution of the stellar mass fraction in galaxies is to plot the ratio of the stellar fraction to the mean relation as a function of redshift $\left(r_{f}(z)=f_{\text {star }}(z) /\left\langle f_{\text {star }}\right\rangle\right)$. Using the same five bins in total mass as above, no trend in $r_{f}(z)$ is evident. However a fit of $r_{f}(z)$ gives a robust upper limit on the evolution over the maximum redshift range $(0-1)$ of $40 \%$. Taking the median redshift of each redshift bin $(0.22,0.72)$, the upper limit on the evolution of the stellar fraction is less than $20 \%$. This number is consistent with the upper limit on the evolution of the relation between total star fraction and $M_{500}$ given by Balogh et al. (2008). Therefore, we conclude that our data do not support the existence of a significant evolution in the zero point and slope of the $f_{500}^{\text {stars }}$ $M_{500}$ relation between redshifts 0 and 1 .

\subsection{The Total Baryon Mass Fraction}

\subsubsection{The Gas Mass Fraction}

In order to determine the total baryon mass fraction in individual systems, we need to estimate the amount of baryons in the form of hot gas which make the ICM. Unfortunately, this cannot be achieved from most of the existing X-ray observations of the total sample because their signal to noise is insufficient for the purpose. Therefore, we have to resort to an estimate of the mean trend of the gas mass fraction as a function of $M_{500}$ established from an independent sample of well observed groups and clusters at $z \leqslant 0.2$, selected from the samples of Vikhlinin et al. 2006 (V06), Arnaud et al. 2007 (APP07) and Sun et al. 2009 (S08). These authors computed gas mass fractions at $R_{500}$ from hydrostatic mass estimates for 10 (V06), 10 (APP07) and 21 (S08, including the best quality tiers 1 and 2 systems) clusters and groups, respectively. The combined sample contains 41 systems and spans the total mass range $1.5 \times 10^{13}-1.1 \times$ $10^{15} M_{\odot}$. After conversion to a common cosmology, a fit of the 

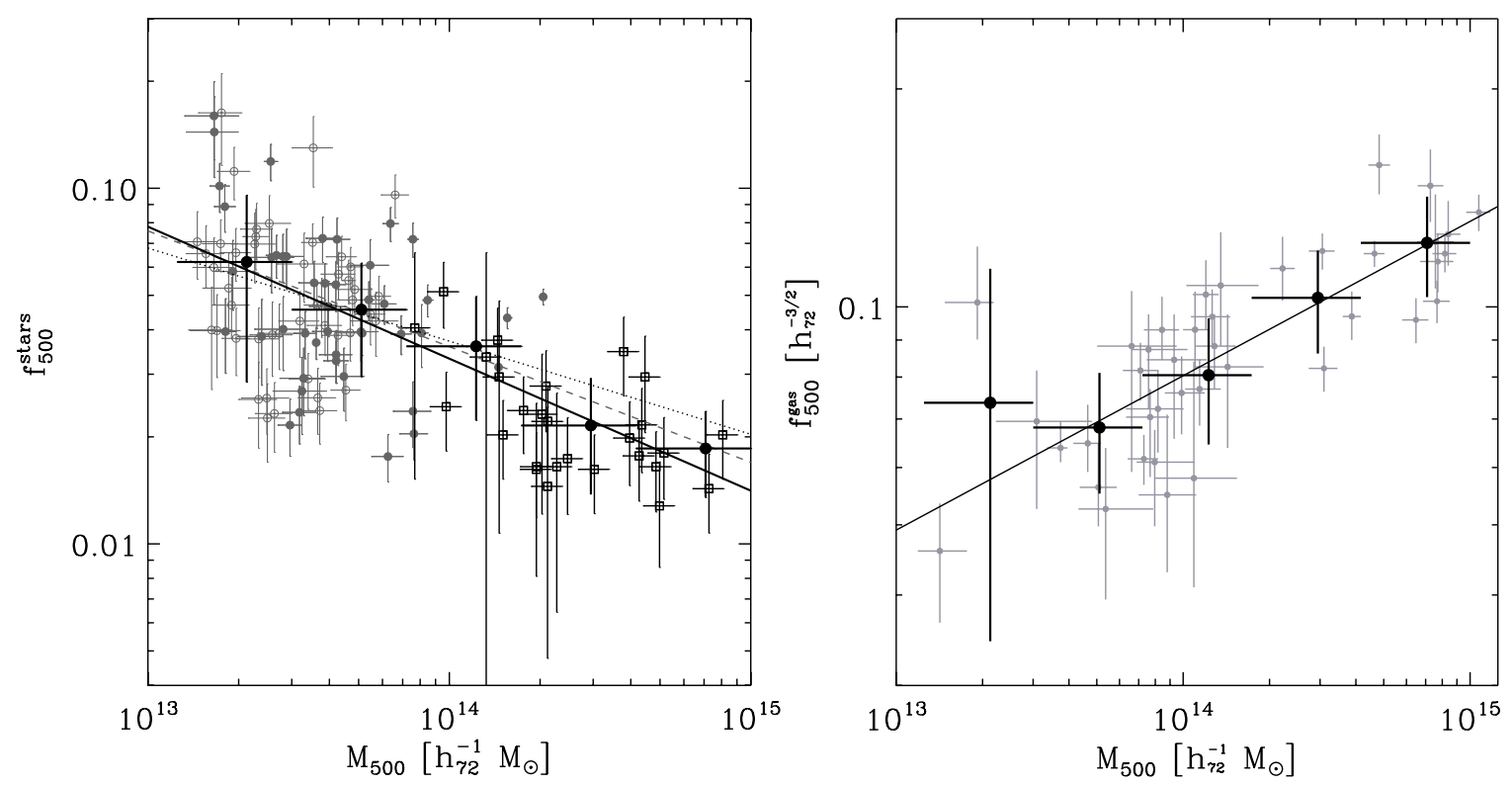

Figure 5. Left panel: stellar-to-total mass ratio vs. total mass for the combined sample of 91 COSMOS X-ray-selected groups (same symbols as in Figure 4) plus 27 nearby clusters of LMS03 (empty squares). The dashed line represents the best-fit relation derived for flag $=1$ groups of the COSMOS sample and the dotted line represents the fit to all COSMOS groups. The solid line shows the best fit relation for all COSMOS groups plus local clusters. All fits are derived taking into account uncertainties in both quantities and the intrinsic scatter in the relation. The ensuing fit parameters are given in Table 1. The large points with error bars show the bi-weight mean and standard deviation of these data binned in five logarithmic bins in total mass. Right panel: gas fraction as a function of the system mass from a combined sample of 41 clusters and groups (Vikhlinin et al. 2006, V06; Arnaud et al. 2007, APP07; Sun et al. 2009, S08). The solid line is the best fit relation $f_{500}^{\mathrm{gas}} \propto M_{500}^{0.2}$. The large points with error bars show the mean and standard deviation of these data binned in five bins of total mass.

combined data set using the WLSS regression yields

$$
f_{500}^{\mathrm{gas}}(h / 0.7)^{3 / 2}=\left(9.3_{-0.2}^{+0.2}\right) \times 10^{-2}\left(\frac{M_{500}}{2 \times 10^{14} M_{\odot}}\right)^{0.21 \pm 0.03},
$$

with a scatter of $17 \%$ about the best-fitting regression line. The data and resulting fit are shown in Figure 5 (fit parameters are given in Table 1). As discussed in the introduction, we assume that this relation is not evolving, in the absence of observations to the contrary. To better elucidate trends with total mass, we divided the data set into the same logarithmic bins in total mass as for the stellar mass fraction, and computed the mean and standard deviation of the distribution of the gas mass fraction values in each bin. The large points with error bars show the trend of these binned data with total mass. The observed relation suggests that lower mass systems have proportionally less gas than high mass systems. Further discussion is available in Pratt et al. (2009).

\subsubsection{The Baryon Mass Fraction (in Galaxies and ICM)}

We now combine the results on the stellar and gas mass fractions derived in the previous two sections to investigate the behavior of the baryonic mass fraction as a function of total mass. At this stage, no contribution is considered from the ICL as defined in Section 4.4.3. In each logarithmic mass bin, we sum the mean contribution from stellar and ICM mass components. The values of $f_{500}^{\text {stars }}$ and $f_{500}^{\text {gas }}$ in each bin are shown in Table 2. As we wish to determine the behavior of the average systems in a given mass bin, for each component the uncertainty is calculated from the standard deviation of the mean (the standard deviation divided by $\sqrt{N-1}$, where $N$ is the number of data points in the bin). The uncertainty on the total baryon mass content is then estimated from the quadratic sum of the individual uncertainties for the stellar and ICM contributions. Figure 6 (lower panel) reproduces the average behavior of the sum of the two baryonic
Table 1

Best-Fit Parameters for the Relation Between Stellar Mass Fraction and Total Mass (Equations (10) and (11)) for Three Samples Considered

\begin{tabular}{lcc}
\hline \hline \multicolumn{1}{c}{ Sample } & $\log (N)$ & $\alpha$ \\
\hline COSMOS flag $=1$ & $-1.35 \pm 0.01$ & $-0.33 \pm 0.12$ \\
COSMOS flag $=1+2$ & $-1.35 \pm 0.01$ & $-0.26 \pm 0.09$ \\
COSMOS+LM03 & $-1.37 \pm 0.01$ & $-0.37 \pm 0.04$ \\
\hline
\end{tabular}

Note. Data were fitted with a power-law $f_{500}^{\text {stars }}=N\left(M_{500} / 5 \times 10^{13} M_{\odot}\right)^{\alpha}$.

Table 2

Measured Values for $f_{500}^{\text {gas }}$ and $f_{500}^{\text {stars }}$ as in Figure 6

\begin{tabular}{lccc}
\hline \hline$M_{500} /\left[h_{72}^{-1} M_{\odot}\right]$ & $f_{500}^{\text {stars }}$ & $f_{500}^{\text {gas }}$ & $f_{500}^{\text {stars }+ \text { gas }}$ \\
\hline $2.1 \mathrm{e}+13$ & $0.062 \pm 0.005$ & $0.074 \pm 0.028$ & $0.136 \pm 0.028$ \\
$5.1 \mathrm{e}+13$ & $0.045 \pm 0.002$ & $0.068 \pm 0.005$ & $0.113 \pm 0.005$ \\
$1.2 \mathrm{e}+14$ & $0.036 \pm 0.004$ & $0.080 \pm 0.003$ & $0.116 \pm 0.005$ \\
$3.0 \mathrm{e}+14$ & $0.021 \pm 0.002$ & $0.103 \pm 0.008$ & $0.124 \pm 0.009$ \\
$7.1 \mathrm{e}+14$ & $0.019 \pm 0.002$ & $0.123 \pm 0.007$ & $0.141 \pm 0.007$
\end{tabular}

Note. Uncertainties correspond to the standard deviation of the mean (see the text for details).

components estimated in the previous sections (i.e., ICM gas and stars associated with galaxies) as a function of total mass for galaxy systems with $2 \times 10^{13} \leqslant M_{500} \leqslant 8.1 \times 10^{14} M_{\odot}$. The ensuing baryon mass fraction is an increasing function of the system mass,

$$
f_{500}^{\text {stars }+ \text { gas }}=(0.123 \pm 0.003) \times\left(\frac{M_{500}}{2 \times 10^{14} M_{\odot}}\right)^{0.09 \pm 0.03} .
$$

This expression is obtained after excluding the lowest mass point which is affected by an extremely large uncertainty since the corresponding gas fraction is estimated from only two groups. 


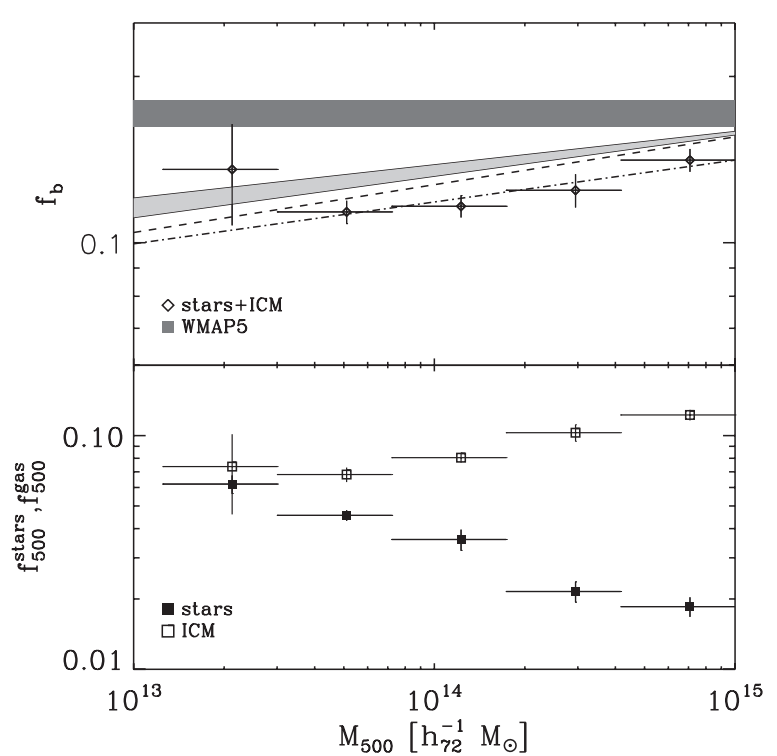

Figure 6. Lower panel: average stellar to dark mass ratio (filled points) for the COSMOS+LM03 sample and average gas fraction (empty points). Uncertainties are computed from the standard deviation of the mean in all cases. Upper panel: total baryonic fraction obtained summing the points in the lower panel compared with the universal value by WMAP5 (dark gray stripe). The dashed-dotted line represents the fit to the measured points. The dashed line represents the fit to the points where the gas fraction has been corrected for a $10 \%$ gas depletion. The light gray stripe is the fit to the relation taking in account both gas depletion and a constant $(11 \%-22 \%)$ ICL contribution to the stellar mass.

\subsection{Comparison with WMAP}

4.4.1. Raw Values

As Figure 6 shows, there is a gap between the values of $f_{500}^{\text {stars+gas }}$ estimated from WMAP5 and those obtained here; this discrepancy, before any correction, is significant at more than $5 \sigma$ for systems less massive than $\sim 10^{14} M_{\odot}$ (see Table 3 ), where the uncertainties are calculated as described in Section 4.3.2.

\subsubsection{Values Corrected for Gas Depletion}

We now correct the value of the baryon fraction for gas depletion. As discussed in Frenk et al. (1999), simulations without feedback suggest that the ICM has a slightly more inflated distribution than the dark matter (see also observations by Pratt \& Arnaud 2002), resulting in a decrease in the gas fraction of $10 \%$ at $R_{500}$. In the absence of indications to the contrary we do not assume a mass dependence for the gas depletion. For average massive clusters $\left(\left\langle M_{500}\right\rangle=7 \times 10^{14} M_{\odot}\right)$ the value of gas depletion-corrected $f_{500}^{\text {stars }+ \text { gastdepl }}$ is consistent within $1.4 \sigma$ with the WMAP5 estimate. However, the gas depletion corrected value in the group regime $\left(\left\langle M_{500}\right\rangle=\right.$ $5 \times 10^{13} M_{\odot}$ ) is still $4.5 \sigma$ discrepant from that of WMAP5. ${ }^{27}$

\subsubsection{Values Corrected for Gas Depletion and ICL}

The existence of a diffuse stellar component in galaxy groups/ clusters is now a well established observational result, but the way the ICL is defined and measured is not unique (see Zibetti 2008 for a recent review). The quality of our observations is insufficient to measure the contribution of diffuse, very low

27 We note that this discrepancy represents a lower limit if a further $10 \%$ reduction of the gas mass is applied due to the clumpiness of the ICM as in Lin et al. (2003). However this correction is not applied in most of the studies of gas component in clusters.
Table 3

Discrepancy of $f_{\mathrm{b}}$ from the WMAP5 Value in Sigma Units

\begin{tabular}{lccc}
\hline \hline$M_{500} /\left[h_{72}^{-1} M_{\odot}\right]$ & $\Delta_{f_{b}} /\left[\sigma_{f_{b}}\right]$ & $\Delta_{f_{b}} /\left[\sigma_{f_{b}}\right]^{\mathrm{a}}$ & $\Delta_{f_{b}} /\left[\sigma_{f_{b}}\right]^{\mathrm{b}}$ \\
\hline $2.1 \mathrm{e}+13$ & $>1.2$ & $>0.8$ & $>0.3$ \\
$5.1 \mathrm{e}+13$ & 5.3 & 4.5 & 3.3 \\
$1.2 \mathrm{e}+14$ & 5.1 & 4.2 & 3.2 \\
$3.0 \mathrm{e}+14$ & 3.7 & 2.6 & 2.1 \\
$7.1 \mathrm{e}+14$ & 2.6 & 1.4 & 1.0 \\
\hline
\end{tabular}

Notes.

${ }^{a}$ After correction for gas depletion.

${ }^{b}$ After correction for gas depletion and ICL.

surface brightness light $\left(>25.8 \mathrm{~K}\right.$ mag $\operatorname{arcsec}^{-2}$ ) within $r_{500}$ directly for individual systems in the sample. To quantify the amount of stellar mass which is associated with diffuse light that escapes detection during the standard photometry extraction with SExtractor (Capak et al. 2007), we are guided by previous observational results. In particular, we consider Zibetti et al. (2005), Krick \& Bernstein (2007), and Gonzalez et al. (2005). Zibetti et al. (2005) used stacking analysis of 683 systems at $z=0.2-0.3$ ranging in total mass from a few times $10^{13}$ to $5 \times 10^{14} M_{\odot}$ (the average total mass is $7 \times 10^{13} M_{\odot}$ ), selected from a $1500 \mathrm{deg}^{2}$ of SDSS-DR1, reaching the unprecedented surface brightness limit of $\sim 32$ mag $\operatorname{arcsec}^{-2}$ ( $R$ band in the $z=0.25$ observed frame). They show that on average the ICL contributes $\sim 11 \%$ of the stellar light within $500 \mathrm{kpc}$. In a complementary study, Krick \& Bernstein (2007) used a sample of massive clusters with a range of morphology, redshift and densities to find that the ICL contributes with $6 \%-22 \%$ to the total cluster light in the $r$ band within one quarter of the virial radius, finding no appreciable correlation with cluster mass. Given these results, we assume that the contribution of the ICL to the total mass of a system is equal to its observed contribution to the total light and ranges between $11 \%$ and $22 \%$. This range is consistent with the theoretical results by Murante et al. (2007) and Purcell et al. (2008), in their attempt of modelling the ICL by numerical simulations. Furthermore, given the complete lack of observational constraints, we assume that the ICL mass fraction is not evolving with redshift for $0<z<1$; this is supported by the simulation of Dubinski et al. (2003) as shown in Feldmeier et al. (2004). We discuss the impact of our choice on the results in Section 4.5. The final gas depletion corrected values including the ICL contribution of $\mathrm{f}_{500}^{\text {stars }+\mathrm{gas}+\mathrm{depl}+\mathrm{ICL}}$ are lower than the WMAP5 estimate across the entire explored mass range; $f_{500}^{\text {stars }+ \text { gas }+ \text { depl+ICL }}$ is in agreement with the WMAP5 result within $1 \sigma$ in the massive cluster regime, but still discrepant at a significance level of at least $3.3 \sigma$ for groups (see Figure 6).

\subsection{Impact of Systematic Effects}

The basic observational result of the present study is that the baryon mass fraction, corrected for gas depletion and ICL contribution, is consistent with WMAP5 estimate within $1 \sigma$ for clusters with $\langle M\rangle=7 \times 10^{14} M_{\odot}$ but is significantly $(3.3 \sigma)$ lower for groups with $\langle M\rangle=5 \times 10^{13} M_{\odot}$. At the cluster scale our result on the baryon fraction is consistent with that of Lin et al. (2003), indicating that different approaches do not show systematic differences in the determination of the gas fraction scaling with the cluster mass. Furthermore, we note that the scaling relation determined by Pratt et al. (2009) is based on three different samples of groups and clusters: this 
should reduce the potential bias produced by sample selection. In Pratt et al. (2009), the best-fit relation to the combined data from hydrostatic estimates reproduces the REXCESS sample distribution where the gas masses have been estimated using the $M-T$ relation of Arnaud et al. (2005). This suggests that potential systematic effects on our estimates of the gas mass fractions at low redshifts are negligible.

In the absence of direct estimates of the gas fraction at $z>0.2$, we have to rely upon the results of existing simulations, which predict the gas fraction within $r_{500}$ to increase on average by $\sim 5 \%$ (adiabatic simulations) or $10 \%-20 \%$ (simulations with cooling and star formation) between $z=0$ and $z=1$ for groups and poor clusters (Kravtsov et al. 2005). Applying a correction to this effect at the median redshift of the COSMOS group sample (by $5 \%-10 \%$ at $z=0.5$ ), the discrepancy in the baryon mass fraction between groups of $\langle M\rangle=5 \times 10^{13} M_{\odot}$ and WMAP5 is reduced to $3.0 \sigma-2.6 \sigma$. Therefore, we conclude that systematic underestimates of the gas fraction alleviate but do not solve the discrepancy at the group scale.

Since inside groups, the stellar mass fraction is comparable to the gas mass fraction, we analyze the impact of the ICL fraction and the adopted stellar $\mathrm{M} / \mathrm{L}$ of the galaxy population. We have adopted a mass independent correction to the total stellar mass fraction for ICL, equal to $11 \%-22 \%$. If a strong anti-correlation between the ICL mass fraction and the total mass of the system exists, and the true ICL mass fraction is equal to $\sim 50 \%$ at the group scale, an agreement between our total baryonic mass fraction and the WMAP5 estimate is reached. Such a figure has been claimed by Gonzalez et al. (2007) for a sample of 23 BCGdominated clusters and groups. However, the ICL-to-BCG light ratio (ICL/BCG) is strongly dependent on the decomposition of the total surface brightness profile of the two components and the photometric depth (Gonzalez et al. 2005; Zibetti 2008). We note that Gonzalez et al. (2005) give ICL/BCG $>5$ by applying a simultaneous decomposition of the surface brightness distribution of BCG+ICL in two De Vaucouleurs components: the outer one is considered as the genuine ICL and the inner one as the BCG. Conversely, Zibetti et al. (2005) obtain ICL/BCG $<0.5$ by fitting only the inner profile with a De Vaucouleurs model (which represents the BCG) and considering all the residual light as ICL. Nevertheless, Zibetti (2008) applied a two-De Vaucouleurs decomposition to the Zibetti et al. (2005) data obtaining ICL/BCG $\sim 2$, and concluded that the ICL+BCG-to-total light ratio is a much more robust measurement, which is likely equal to 0.3 (in light) for systems of average mass $7 \times 10^{13} M_{\odot}$. The high value of ICL/BCG found by Gonzalez et al. (2007) may be the result of a sample bias, as suggested by the same authors. On the other hand, the lack of trends reported by Zibetti et al. (2005) could be intrinsically biased by the adoption of a fixed metric aperture of $500 \mathrm{kpc}$, which correspond to smaller fraction of R200 for more massive clusters. Given the steeper profile of the ICL with respect to galaxies, the ICL fraction of more massive clusters could be overestimated and a correction for this effect could reconcile these results with the negative trend found by Gonzalez et al. (2007), but not with the extreme values of ICL+BCG-to-total light ratio. Generally, it is evident that better determinations of the trends of the ICL with cluster mass and richness are needed.

The COSMOS groups sample contains a whole range of systems, which exhibit a BCG-to-galaxy stellar mass ratio from 0.2 to 0.9 . For these groups, the estimated ICL+BCG-to-total light ratio for the average group is 0.36 , broadly consistent with the generally accepted average value of 0.3 (Gonzalez et al. 2007; Zibetti 2008). This suggests that we are not missing an important contribution of the stellar mass in our analysis, in spite of our definition of ICL.

Another systematic effect may be introduced by the computation of the stellar M/L for the ensemble of the member galaxies and the ICL. In our case, we use M/L values that correspond to the individual star formation histories of individual member galaxies (Arnouts et al. 2007) and we do not make assumption on the $\mathrm{M} / \mathrm{L}$ of the ICL. Hence, the major source of systematics on the stellar M/L of our galaxies is given by the adopted initial mass function (IMF). For instance, a change from a standard Salpeter to a Chabrier IMF reduces the M/L by $30 \%$ (Longhetti $\&$ Saracco 2009). This translates into a decrease by $30 \%$ of the stellar mass associated with galaxies which makes the bulk of the total stellar mass in our systems. There is no compelling reason to abandon the Salpeter IMF (Renzini 2005), but it is a possibility explored in the literature. Lin et al. (2003) obtained the stellar M/L for the ensemble of group/cluster member galaxies by folding in a morphological type dependent $\mathrm{M} / \mathrm{L}$ with the temperature dependence of the spiral fraction; Gonzalez et al. (2007) assumed that the ICL and all member galaxies share the same stellar $\mathrm{M} / \mathrm{L}$, as the one that characterize an earlytype galaxy. The latter case assumes that the intergalactic stars are homogeneous with the BCG stellar population. However, it has been suggested that the ICL may (also) origin from the stripping of non-BCG galaxies inside the group/cluster (Purcell et al. 2008; Pierini et al. 2008), which are on average bluer than the BCG, especially in groups (Zabludoff \& Mulchaey 1998; Weinmann et al. 2006; Poggianti et al. 2006). For example, if the ICL M/L used in Gonzalez et al. (2007) is overestimated by a factor 2 , it translates in the systematic overestimation of the baryon mass fraction by $10 \%$.

This systematic effect has the same amplitude, but opposite sign, of the potential offset applied to the gas fraction-mass relation according to Gonzalez et al. (2007). Therefore, we conclude that a $3 \sigma$ discrepancy between the baryon mass fraction of groups and the WMAP value holds against major systematic effects on the stellar populations either diffuse or associated with galaxies.

An overestimate of the total M/L is not enough to explain the values of the stellar mass fraction for the lowest mass systems in Gonzalez et al. (2007), which largely exceed the constraint on the total baryon fraction set by WMAP5 (as also noted in Balogh et al. 2008). A way out is a systematic and large underestimate of the total masses of these systems, as also suggested by Balogh et al. (2008). ${ }^{28}$

We conclude that a robust estimate of the total mass is crucial for systems with the lowest mass (in our sample $\left\langle M_{500}\right\rangle \sim$ $\left.2 \times 10^{13} M_{\odot}\right)$. Our estimates are based on the $L_{\mathrm{X}}-M_{200}$ relation established via the weak lensing analysis in A. Leauthaud et al. (2009, in preparation), and exhibit a typical uncertainty of $30 \%$. The use of different total mass estimators could offer a test of the presence of systematics, but unfortunately this is still hard to achieve for statistical large samples of groups at different redshifts.

\section{DISCUSSION}

We have investigated if the discrepancy between estimates of the total baryon mass fraction obtained from observations of the CMB and of galaxy groups persists when a large, unbiased sample of well characterized groups is considered.

\footnotetext{
28 These objects certainly impact the strongly inverse total mass dependence
} of the total stellar mass fraction found by Gonzalez et al. (2007). 
The COSMOS $2 \mathrm{deg}^{2}$ survey meets this requirement, yielding 91 candidate X-ray groups/poor clusters at redshift $0.1 \leqslant z \leqslant 1$. In order to extend the span in total mass to $\mathrm{t} 2$ orders of magnitude $\left(2 \times 10^{13}<M_{500}<1.2 \times 10^{15} M_{\odot}\right)$, we consider 27 nearby clusters investigated by Lin et al. (2003). Comparable robust measurements of total mass and total stellar mass (in galaxies) exist for individual objects of both subsamples, as shown in the previous sections. In addition, the same scaling relation is used to estimate the gas mass fraction in both subsamples. This enables us to build a joint sample of $118 \mathrm{X}$-ray-selected groups and clusters at $z \leqslant 1$ for which the importance of systematics is reduced (see Section 2). For this sample, the behavior of the total stellar mass fraction as a function of the total mass can be investigated for a large range in total mass and, for the first time, in redshift (at least for groups). The results of our analysis and their impact on the widely accepted paradigm of the hierarchical growth of structure in the universe are discussed hereafter.

\subsection{The Stellar Mass Fraction}

We have shown (Figure 5) that the stellar-to-total mass ratio in COSMOS groups and in 27 local clusters is anticorrelated with the total mass of the system. This relation is given by $f_{500}^{\text {stars }} \propto M_{500}^{-0.37 \pm 0.04}$, which holds also after introducing the mass independent correction for the ICL (see Section 4.4). The global trend between $f_{500}^{\text {stars }}$ and $M_{500}$ is consistent with that observed in clusters at $z<0.3$ both by LMS03 and Laganá et al. (2008) using much smaller samples. We extend their results to the low mass regime by one decade and to higher redshift.

The difference in the number of stars formed per unit of halo mass between groups and clusters has been interpreted in terms of a varying efficiency of the star formation with the total mass of the system (e.g., Lin et al. 2003). A variation in the star formation efficiency for systems with virial temperatures $\geqslant 10^{7} \mathrm{~K}$ is a result of simulations by Springel \& Hernquist (2003); it is interpreted in terms of cooling flows being less efficient in shutting off star formation in groups. An alternative possibility is that clusters are formed not only by merging of groups and smaller clusters but also that they accrete a large fraction of their galaxies (with a low stellar mass fraction, of the order of 0.01) from the field (White \& Frenk 1991; Marinoni \& Hudson 2002). However, after a mass independent correction for the ICL contribution (introduced in Section 4.4), the relation $f_{500}^{\text {stars }} \propto M_{500}^{-0.37 \pm 0.04}$ is in agreement with the constraint on the slope set by the hierarchical model of structure formation under the assumption that at least half of the stars in groups were formed by $z=1$ (Balogh et al. 2008). ${ }^{29}$ This is supported by the apparent absence of evolution for this relation in our sample within the redshift range $0.1-1$. This shows how observational studies such as the present one can improve the constraints on models and foster our understanding of the underlying physical processes.

\subsection{The Total Baryon Mass Fraction}

Combining the computed stellar mass fraction with the estimated gas mass fraction derived from the mean local relation in Pratt et al. (2009), we find that the gas plus stellar (galaxies) baryon mass fraction increases by $\sim 25 \%$ (from $\sim 0.11$ to $\sim 0.14$ ) when the total mass increases by a factor of 100 . After a constant $10 \%$ correction for gas depletion and a further

\footnotetext{
${ }^{29}$ We note that a steeper relation is obtained when the strongly inverse mass dependent ICL fraction of Gonzalez et al. (2007) is used (see Balogh et al. (2008) for the discussion).
}

correction for a constant $11 \%-22 \%$ ICL contribution, the value of $f_{500}^{\text {stars+gas+depl+ICL }}$ for an average cluster is consistent within $1 \sigma$ with the cosmic value measured by WMAP, while the $f_{500}^{\text {stars+gas+depl+ICL }}$ found for an average group differs from it at more than $3 \sigma$. Given the heterogeneity of the sample (see e.g., Figure 5), for some objects the gap between $f_{500}^{\text {stars+gas+depl+ICL }}$ and the WMAP5 value could be negligible or, conversely, statistically more significant for objects in the same bin of total mass, but at the two extremes of the distribution in $f_{\text {stars }}^{500}$. Unfortunately, we do not have a measure of the gas mass fraction for individual objects, therefore we focus on the behavior of the average object. We did likewise for the ICL by assuming a fixed fractional contribution of $11 \%-22 \%$ across the entire mass range. Possible systematic effects introduced by our definition and estimate of the ICL contribution are discussed in Section 4.5. Here, we stress that they do not lead to an anomalously low $\mathrm{BCG}+\mathrm{ICL}$ contribution to the total mass of the system. Thus, the discrepancy at the groups regime in not erased by uncertainties on the stellar mass fraction. In the absence of evidence for a systematic and relevant underestimation of the gas mass fraction in our systems (see Section 4.5), we interpret the discrepancy as a lack of gas, by $33 \%$, at the group regime. This may be produced by feedback (stellar and/or AGN), as suggested by high-resolution cosmological simulations including cooling, star formation, supernova feedback, and AGN radio-mode feedback in galaxy clusters and groups (Puchwein et al. 2008, Bower et al. 2008, Short \& Thomas 2008). Since supernova feedback appears to be insufficient to explain the $L_{X}-T$ relation (Puchwein et al. 2008), feedback by AGN seems necessary. According to this interpretation, gas can be removed from within $R_{500}$ mainly as a consequence of the mechanical heating produced by a central AGN. The action of the AGN is larger in groups than in clusters simply because the potential well is shallower in the former systems. In a forthcoming work, we will quantify the feedback by AGN radio-mode for the COSMOS groups. Another proposed mechanism capable of accounting for the "missing" gas is "filamentary heating" (Voit \& Bryan 2001). Low entropy gas is consumed in star formation before the group formation, which eventually raises the entropy of the gas which becomes the ICM. The resulting higher entropy level inhibits the gas from falling toward the center of the potential well, which can explain the lack of gas in the central region of groups (Sun et al. 2009).

\section{CONCLUSIONS}

The baryon mass fraction is a parameter which can be constrained by the primordial light elements abundance set by the nucleosynthesis at early epochs. It can be independently measured from observations of the CMB (e.g., WMAP) or of galaxy groups/clusters. Different studies of the baryon mass fraction in nearby galaxy systems have reported values lower than the one from WMAP, the discrepancy being larger for groups than clusters. We investigate if this discrepancy persists when a sample of local clusters is supplemented by a large, unbiased sample of groups at $0.1 \leqslant z \leqslant 1.0$. Hereafter, we list our conclusions.

1. The stellar mass fraction associated with galaxies is anti-correlated with the mass of the system: $f_{500}^{\text {stars }} \propto$ $M_{500}^{-0.37 \pm 0.04}$. This is consistent with previous results on local clusters. The validity of this result is now extended by one decade in total mass and to redshift 1. 
2. The previous relation holds after correcting the stellar mass fraction for a mass independent $11 \%-22 \%$ contribution from the ICL as suggested by both observations and simulations. The slope of the $f_{500}^{\text {stars }}-M_{500}$ relation is consistent with the constraint set by the hierarchical paradigm of structure formation (Balogh et al. 2008). No significant evolution in the relation between $f_{500}^{\text {stars }}$ and $M_{500}$ is observed. This supports the scenario in which massive clusters form mostly by merging of less massive groups and clusters, and observed groups in the redshift range $0-1$ have formed the bulk of their stellar mass by $z \sim 1.0$.

3. Combining measured values of the stellar mass fraction with values of the gas mass fraction estimated from an average relation obtained for a local sample, $f_{500}^{\text {stars+gas }}$ increases by $25 \%$ from groups to clusters. After the introduction of appropriate corrections for gas depletion and ICL contribution, the total baryonic mass fraction at the groups regime still differs from the WMAP5 value at $3.3 \sigma$. We interpret the origin of this discrepancy as a lack of gas (by 33\%), which can be produced either by feedback (supernovae and/or radio-mode AGN heating) or by "filamentary heating."

Our results provide useful constraints on simulations of the aforementioned processes. In particular, the availability of a large unbiased sample of groups offers direct and stringent constraints on models rather than relying on extrapolation of the behavior of the stellar fraction as a function of mass in the entire family of systems with $10^{13}<M_{500}<10^{14} M_{\odot}$. Future observations will increase both the statistics and the redshift sampling rate, so that a test and extension of our conclusions will be possible.

The authors thank the anonymous referee for her/his valuable comments, which led to a significant improvement of the paper. We acknowledge the contributions of the entire COSMOS collaboration; more information on the COSMOS survey are available at http://www.astr.caltech.edu/ cosmos. This research was supported by the DFG Cluster of Excellence Origin and Structure of the Universe (http://www.universe-cluster.de). D.P. acknowledges support by the German Deutsches Zentrum für Luft- und Raumfahrt, DLR project number 50 OR 0405.

\section{REFERENCES}

Allen, S. W., Schmidt, R. W., Ebeling, H., Fabian, A. C., \& van Speybroeck, L. 2004, MNRAS, 353, 457

Arnaud, M., Pointecouteau, E., \& Pratt, G. W. 2005, A\&A, 441, 893

Arnaud, M., Pointecouteau, E., \& Pratt, G. W. 2007, A\&A, 474, L37

Arnouts, S., et al. 2002, MNRAS, 329, 355

Arnouts, S., et al. 2007, A\&A, 476, 137

Balogh, M. L., McCarthy, I. G., Bower, R. G., \& Eke, V. R. 2008, MNRAS, 385, 1003

Bartelmann, M. 1996, A\&A, 313, 697

Beers, T. C., Flynn, K., \& Gebhardt, K. 1990, AJ, 100, 32

Bialek, J. J., Evrard, A. E., \& Mohr, J. J. 2001, ApJ, 555, 597

Biviano, A., \& Salucci, P. 2006, A\&A, 452, 75

Bolzonella, M., et al. 2009, arXiv:0907.0013

Bower, R. G., McCarthy, I. G., \& Benson, A. J. 2008, MNRAS, 390, 1399

Bundy, K., Ellis, R. S., \& Conselice, C. J. 2005, ApJ, 625, 621

Capak, P., et al. 2007, ApJS, 172, 99

David, L. P., Arnaud, K. A., Forman, W., \& Jones, C. 1990, ApJ, 356, 32

Diehl, S., \& Statler, T. S. 2007, ApJ, 668, 150

Dubinski, J., Koranyi, D., \& Geller, M. 2003, in IAU Symp. 208, Astrophysical

Supercomputing using Particle Simulations, ed. J. Makino \& P. Hut (San

Francisco, CA: ASP), 237

Dunkley, J., et al. 2009, ApJS, 180, 306

Elvis, M. \& C-COSMOS Team 2006, BAAS, 38, 1006

Ettori, S. 2003, MNRAS, 344, L13

Evrard, A. E. 1997, MNRAS, 292, 289
Feldmeier, J. J., Mihos, J. C., Morrison, H. L., Harding, P., Kaib, N., \& Dubinski, J. 2004, ApJ, 609, 617

Finoguenov, A., et al. 2007, ApJS, 172, 182

Frenk, C. S., et al. 1999, ApJ, 525, 554

Gilfanov, M., Grimm, H.-J., \& Sunyaev, R. 2004, MNRAS, 351, 1365

Gonzalez, A. H., Zabludoff, A. I., \& Zaritsky, D. 2005, ApJ, 618, 195

Gonzalez, A. H., Zaritsky, D., \& Zabludoff, A. I. 2007, ApJ, 666, 147

Grimm, H.-J., Gilfanov, M., \& Sunyaev, R. 2003, MNRAS, 339, 793

Guzzo, L., et al. 2007, ApJS, 172, 254

Hasinger, G., et al. 2007, ApJS, 172, 29

He, P., Feng, L.-L., \& Fang, L.-Z. 2006, ApJ, 623, 601

Hoekstra, H. 2007, MNRAS, 379, 317

Ilbert, O., et al. 2009, ApJ, 690, 1236

Koekemoer, A. M., et al. 2007, ApJS, 172, 196

Komatsu, E., et al. 2009, ApJS, 180, 330

Kravtsov, A. V., Nagai, D., \& Vikhlinin, A. A. 2005, ApJ, 625, 588

Krick, J. E., \& Bernstein, R. A. 2007, AJ, 134, 466

Küpcü Yoldaş, A., et al. 2007, A\&A, 463, 893

Laganá, T. F., Lima Neto, G. B., Andrade-Santos, F., \& Cypriano, E. S. 2008, A\&A, 485, 633

Lilly, S. J., et al. 2007, ApJS, 172, 70

Lin, Y.-T., \& Mohr, J. J. 2004, ApJ, 617, 879

Lin, Y.-T., Mohr, J. J., \& Stanford, S. A. 2003, ApJ, 591, 749

Longhetti, M., \& Saracco, P. 2009, MNRAS, 394, 774

Marinoni, C., \& Hudson, M. J. 2002, ApJ, 569, 101

Massey, R., et al. 2007, Nature, 445, 286

McCarthy, I. G., Bower, R. G., \& Balogh, M. L. 2007, MNRAS, 377, 1457

Moretti, A., et al. 2004, A\&A, 428, 21

Murante, G., Giovalli, M., Gerhard, O., Arnaboldi, M., Borgani, S., \& Dolag, K. 2007, MNRAS, 377, 2

Navarro, J. F., Frenk, C. S., \& White, S. D. M. 1997, ApJ, 490, 493 (NFW)

Pannella, M., et al. 2006, ApJ, 639, L1

Pierini, D., Zibetti, S., Braglia, F., Böhringer, H., Finoguenov, A., Lynam, P. D., \& Zhang, Y.-Y. 2008, A\&A, 483, 727

Poggianti, B. M., et al. 2006, ApJ, 642, 188

Pratt, G. W., \& Arnaud, M. 2002, A\&A, 394, 375

Pratt, G. W., Arnaud, M., \& Pointecouteau, E. 2006, A\&A, 446, 429

Pratt, G. W., Croston, J. H., Arnaud, M., \& Böhringer, H. 2009, A\&A, 498, 361

Puchwein, E., Sijacki, D., \& Springel, V. 2008, ApJ, 687, L53

Purcell, C. W., Bullock, J. S., \& Zentner, A. R. 2008, MNRAS, 391, 550

Renzini, A. 2005, in ASSL, Vol. 327, The Initial Mass Function 50 Years Later, ed. E. Corbelli \& F. Palle (Dordrecht: Springer), 221

Rosati, P., della Ceca, R., Norman, C., \& Giacconi, R. 1998, ApJ, 492, L21

Rykoff, E. S., et al. 2008, MNRAS, 387, L28

Sanders, D. B., et al. 2007, ApJS, 172, 86

Sales, L. V., Navarro, J. F., Lambas, D. G., White, S. D. M., \& Croton, D. J. 2007, MNRAS, 382, 1901

Salpeter, E. E. 1955, ApJ, 121, 161

Salvato, M., et al. 2009, ApJ, 690, 1250

Sandage, A. 1986, A\&A, 161, 89

Schechter, P. 1976, ApJ, 203, 297

Scoville, N., et al. 2007, ApJS, 172, 1

Scoville, N., et al. 2007, ApJS, 172, 150

Short, C. J., \& Thomas, P. A. 2008, arXiv:0811.3166

Springel, V., \& Hernquist, L. 2003, MNRAS, 339, 312

Sun, M., Voit, G. M., Donahue, M., Jones, C., Forman, W., \& Vikhlinin, A. 2009, ApJ, 693, 1142

Taniguchi, Y., et al. 2007, ApJS, 172, 9

Vikhlinin, A., Kravtsov, A., Forman, W., Jones, C., Markevitch, M., Murray, S S., \& Van Speybroeck, L. 2006, ApJ, 640, 691

Vikhlinin, A., McNamara, B. R., Forman, W., Jones, C., Quintana, H., \& Hornstrup, A. 1998, ApJ, 502, 558

Voit, G. M., \& Bryan, G. L. 2001, Nature, 414, 425

Weinmann, S. M., van den Bosch, F. C., Yang, X., \& Mo, H. J. 2006, MNRAS, 366,2

White, S. D. M., \& Frenk, C. S. 1991, ApJ, 379, 52

White, S. D. M., Navarro, J. F., Evrard, A. E., \& Frenk, C. S. 1993, Nature, 366, 429

Wilkins, S. M., Trentham, N., \& Hopkins, A. M. 2008, MNRAS, 385, 687

Zabludoff, A. I., \& Mulchaey, J. S. 1998, ApJ, 496, 39

Zamojski, M. A., et al. 2007, ApJS, 172, 468

Zhao, D. H., Jing, Y. P., Mo, H. J., \& Boerner, G. 2008, arXiv:0811.0828

Zibetti, S. 2008, in IAU Symp. 244, Dark Galaxies and Lost Baryons, ed. J. I. Davies \& M. J. Disney (Cambridge: Cambridge Univ. Press), 176

Zibetti, S., White, S. D. M., Schneider, D. P., \& Brinkmann, J. 2005, MNRAS, 358,949 